This document is confidential and is proprietary to the American Chemical Society and its authors. Do not copy or disclose without written permission. If you have received this item in error, notify the sender and delete all copies.

\title{
Acene Ring Size Optimization in Fused Lactam Polymers Enabling High n-Type Organic Thermoelectric Performance
}

\begin{tabular}{|c|c|}
\hline Journal: & Journal of the American Chemical Society \\
\hline Manuscript ID & ja-2020-10365u \\
\hline Manuscript Type: & Article \\
\hline $\begin{array}{r}\text { Date Submitted by the } \\
\text { Author: }\end{array}$ & 28-Sep-2020 \\
\hline Complete List of Authors: & $\begin{array}{l}\text { Chen, Hu; King Abdullah University of Science and Technology, KAUST } \\
\text { Solar Centre } \\
\text { Moser, Maximilian; University of Oxford, Chemistry; Imperial College } \\
\text { London, Chemistry } \\
\text { Wang, Suhao; Linköping University, Dept. of Science and Technology } \\
\text { (ITN) } \\
\text { Jellett, Cameron; Imperial College London, Chemistry } \\
\text { Thorley, Karl; University of Kentucky, } \\
\text { Harrison, George; King Abdullah University of Science and Technology, } \\
\text { KAUST Solar Centre } \\
\text { Jiao, Xuechen; Monash University, Materials Science and Engineering } \\
\text { Xiao, Mingfei; University of Cambridge, Cavendish Laboratory } \\
\text { Purushothaman, Balaji; King Abdullah University of Science and } \\
\text { Technology, KAUST Solar Centre } \\
\text { Alsufyani, Maryam; King Abdullah University of Science and Technology, } \\
\text { KAUST Solar Centre } \\
\text { Bristow, Helen; Imperial College London, Chemistry; University of } \\
\text { Oxford, Chemistry } \\
\text { De Wolf, Stefaan; King Abdullah University of Science and Technology, } \\
\text { Wadsworth, Andrew; University of Oxford, Chemistry; Imperial College } \\
\text { London, Chemistry } \\
\text { McNeill, Christopher; Monash University, Materials Science and } \\
\text { Engineering } \\
\text { Sirringhaus, Henning; University of Cambridge, Cavendish Laboratory } \\
\text { Fabiano, Simone; Linköping University, Dept. of Science and Technology } \\
\text { (ITN) } \\
\text { McCulloch, Iain; University of Oxford, Chemistry; King Abdullah } \\
\text { University of Science and Technology, KAUST Solar Centre }\end{array}$ \\
\hline
\end{tabular}

\section{SCHOLARONE ${ }^{\text {m }}$ Manuscripts}




\section{Acene Ring Size Optimization in Fused Lactam Polymers Enabling High n-Type Organic Thermoelectric Performance}

Hu Chen, ${ }^{1 \dagger^{* *}}$ Maximilian Moser, ${ }^{2,3{ }^{* *}}$ Suhao Wang, ${ }^{4}$ Cameron Jellett, ${ }^{2}$ Karl Thorley, ${ }^{5}$ George T. Harrison, ${ }^{1}$ Xuechen Jiao, ${ }^{6}$ Mingfei Xiao, ${ }^{7}$ Balaji Purushothaman, ${ }^{1}$ Maryam A. Alsufyani, ${ }^{1}$ Helen Bristow, ${ }^{2,3}$ Stefaan De Wolf, ${ }^{1}$ Andrew Wadsworth, ${ }^{2,3}$ Christopher R. McNeill, ${ }^{6}$ Henning Sirringhaus, ${ }^{7}$ Simone Fabiano ${ }^{4}$ and Iain McCulloch ${ }^{1,3}$

${ }^{1}$ King Abdullah University of Science and Technology (KAUST), Physical Science and Engineering Division, Thuwal, 23955-6900, Saudi Arabia

${ }^{2}$ Imperial College London, Department of Chemistry and Centre for Plastic Electronics, London, W12 0BZ, United Kingdom

${ }^{3}$ University of Oxford, Department of Chemistry, Oxford, OX1 3TA UK

${ }^{4}$ Laboratory of Organic Electronics, Department of Science and Technology, Linköping University, Norrköping, SE-60174, Sweden

${ }^{5}$ University of Kentucky, Department of Chemistry, Lexington (KY), 40506-0055, USA

${ }^{6}$ Monash University, Department of Materials Science and Engineering, Wellington Road, Clayton, Victoria, 3800, Australia

${ }^{7}$ University of Cambridge, Department of Physics, Cambridge, CB2 1TN, United Kingdom

$\dagger$ These authors contributed equally to this work.

E-mail: hu.chen@kaust.edu.sa; maximilian.moser13@imperial.ac.uk 


\begin{abstract}
Three n-type fused lactam semiconducting polymers were synthesized for thermoelectric and transistor applications via a cheap, highly atom-efficient and non-toxic transition-metal free aldol polycondensation. Energy level analysis of the three polymers demonstrated that reducing the central acene core size from two anthracene (A-A), to mixed naphthalene-anthracene (A-N) and two naphthalene cores $(\mathbf{N}-\mathbf{N})$ resulted in progressively larger electron affinities, thereby leading to an increasingly more favorable and efficient solution doping process when employing 4-(2,3-dihydro-1,3-dimethyl-1H-benzimidazol-2-yl)N,N-dimethylbenzenamine (N-DMBI) as the dopant. Meanwhile, organic field effect transistor (OFET) mobility data showed the $\mathbf{N}-\mathbf{N}$ and $\mathbf{A}-\mathbf{N}$ polymers to feature the highest charge carrier mobilities, further highlighting the benefits of aryl core contraction to the electronic performance of the materials. Ultimately, the combination of these two factors resulted in $\mathbf{N}-$ $\mathbf{N}, \mathbf{A}-\mathbf{N}$ and A-A to display power factors (PF) of $3.2 \mu \mathrm{W} \mathrm{m} \mathrm{m}^{-1} \mathrm{~K}^{-2}, 1.6 \mu \mathrm{W} \mathrm{m} \mathrm{m}^{-1} \mathrm{~K}^{-2}$ and $0.3 \mu \mathrm{W}$ $\mathrm{m}^{-1} \mathrm{~K}^{-2}$ respectively when doped with N-DMBI, whereby the PF recorded for $\mathbf{N}-\mathbf{N}$ and $\mathbf{A}-\mathbf{N}$ are amongst the highest reported in the literature for n-type polymers. Importantly, the results reported in this study highlight that modulating the size of the central acene ring is a highly effective molecular design strategy to optimize the thermoelectric performance of conjugated polymers thus also providing new insights into the molecular design guidelines for the next generation of high-performance n-type materials for thermoelectric applications.
\end{abstract}

\title{
Introduction
}

With the continuously growing demand for energy and the need to reduce greenhouse gas emissions it is imperative to develop sustainable and environmentally friendly energy solutions in order to effectively combat global warming. Given the ubiquity of waste heat produced from industrial processes, thermoelectric generators capable of converting thermal gradients into electrical energy are a promising option to contribute solving the energy issues 
faced in the $21^{\text {st }}$ century. ${ }^{1}$ Thermoelectric generators are particularly attractive since they do not contain any moving mechanical components, thus reducing wear and improving the durability of devices. ${ }^{2}$ Moreover, they can be easily scaled by simply increasing the number and/or size of the legs within a generator, hence allowing their applicability to a range of systems, including sensors, electronic components for the internet of things (IoT) and wearable electronics. ${ }^{1,3,4}$ Although the vast majority of thermoelectric research has been focused on the use of inorganic materials, e.g. bismuth chalcogenides, half Heusler alloys, skutterutides, ${ }^{5}$ their organic counterparts display several advantages, including: (i) their compatibility with solution-based processing and consequently also low-cost roll-to-roll printing techniques; (ii) their reduced toxicity; (iii) their lightweight and flexible nature, enabling new applications, (iv) the significantly higher abundance and lower cost of their constituent elements and (v) their lower thermal conductivity. ${ }^{1,2,6,7}$ Nonetheless, organic materials also display various drawbacks, including their reduced thermal stability, confining their use to the low temperature regime (operating temperatures $<200{ }^{\circ} \mathrm{C}$ ) and lower thermoelectric performance. The development of higher performing organic thermoelectric (OTE) materials is thus imperative to promote their wider adoption.

Thermoelectric performance is described by the dimensionless figure of merit ZT, which mathematically is expressed as follows.

$$
Z T=\frac{\sigma S^{2} T}{\kappa}
$$

where $\sigma$ is the electrical conductivity, $S$ the Seebeck coefficient (i.e. the thermopower), $T$ the temperature and $\kappa$ the thermal conductivity. 
Due to the difficulty of measuring $\kappa$, the thermoelectric performance of a specific material is often simplified and reduced to its power factor (PF) given in equation 2.

$$
P F=\sigma S^{2}
$$

While electrical conductivities $>1000 \mathrm{~S} \mathrm{~cm}^{-1}$ and PFs $>100 \mu \mathrm{W} \mathrm{m}^{-1} \mathrm{~K}^{-2}$ have been achieved for several p-type organic semiconductors, ${ }^{8-11}$ even the highest performing n-type OTE polymers lag far behind these values. ${ }^{12-15}$ This performance disparity presents a significant issue, since maximizing the energy conversion process in a thermoelectric generator necessitates a balanced performance of both p- and n-type components. Developing new n-type polymers with improved thermoelectric performances and formulating structure-property relationships are therefore of utmost importance.

So far, two main classes of materials for n-type OTEs have been developed, one based on donor-acceptor (D-A) polymers and one based on all-acceptor ladder-type polymers. The most widely explored D-A polymers for OTE to date are naphthalenetetracarboxylic diimide (NDI) based polymers, including the well-studied N2200. In thermoelectric devices, N2200 affords electrical conductivities in the range of $10^{-3} \mathrm{~S} \mathrm{~cm}^{-1}$ and PFs in the order of $10^{-2} \mu \mathrm{W} \mathrm{m}-$ ${ }^{1} \mathrm{~K}^{-2} \cdot{ }^{16-20}$ The modest performance of $\mathrm{N} 2200$ is typically ascribed to: (i) the poor miscibility between N2200 and the employed dopant ${ }^{19,21}$ and (ii) the strong localization of its lowest unoccupied molecular orbital (LUMO), and hence the polaron on the NDI fragment, which arises from a combination of the large dihedral angle between the NDI and bithiophene (T2) moieties and the poor-electron accepting abilities of the T2 unit. Since previous studies have suggested a direct relationship between polaron delocalization and macroscopic conductivity, the above findings explain the poor performance of N2200 in OTEs. ${ }^{16,18}$ The general unsuitability of the D-A approach for OTEs has also been demonstrated in a related set of 
studies on p-type OTE materials, in which a D-A polymer backbone was compared to its alldonor analogue. ${ }^{22}$ The results obtained indicate that the D-A structure poses an obstacle to efficient doping, with the acceptor moiety being inactive for p-type doping. In an analogous fashion, one can envisage that this concept is likely to also apply to the case when D-A polymers are employed as n-type OTE materials, where the donor units will be inactive for ntype doping. Nonetheless, a few high-performance D-A materials for OTE applications have been successfully developed, with molecular design strategies focusing primarily on increasing the electron withdrawing nature of the donor co-monomer and reducing the dihedral angles across the polymer backbone. ${ }^{12-14,17,23}$ For example, one strategy that was successfully employed to overcome the aforementioned limitations posed by N2200, involved substitution of the T2 comonomer with a bithiazole (Tz2). ${ }^{17}$ The advantages of using the Tz2 unit were two-fold: not only did the Tz2 unit promote polymer backbone planarity by minimizing steric repulsions with the NDI unit, but its more electron-withdrawing nature compared to bithiophene, extended the LUMO over the full backbone, consequently also promoting the delocalization of the polaron. Overall, this resulted in the Tz2 containing polymer to have a more than 30-times larger $\sigma$ compared to N2200, which was also reflected in its improved PF of $1.5 \mu \mathrm{W} \mathrm{m} \mathrm{m}^{-1} \mathrm{~K}^{-2}$.

Conjugated ladder-type polymers can also exhibit an all-acceptor polymer backbone, with several design features that render them particularly attractive for n-type OTE: (i) conjugated ladder polymers frequently display excellent thermal stability, thus rendering them ideal candidates for thermoelectric applications, where thermal stability is crucial. ${ }^{15,18,24,25}$ (ii) The presence of electron-withdrawing carbonyl groups in the conjugated polymer backbone is beneficial as they contribute to reducing the LUMO energy, thus rendering the n-type doping of these materials thermodynamically more favourable. ${ }^{15,25,26}$ (iii) Their highly rigid 
conjugated polymer backbones facilitate polaron delocalization, consequently aiding charge carrier transport. ${ }^{18}$

Although the performance of ladder type polymers for n-type thermoelectric applications have improved considerably in recent times, the elucidation of structure-property relationships of conjugated ladder-type polymers for n-type OTE has remained scarce in the literature, with only two studies evaluating the effects of rational chemical modification in terms of device performance. ${ }^{15,25}$ To provide further insights into the design of highperformance n-type OTE semiconductors we have investigated the modulation of the aromatic core size of a fused lactam polymer backbone to tune the thermoelectric performance. This involved synthesizing three fused acene containing polymers formed by the aldol condensation of bis-isatin and bis-oxindole monomers, specifically $\mathbf{N}-\mathbf{N}, \mathbf{A}-\mathbf{N}$ and $\mathbf{A}-\mathbf{A}$, with their structures illustrated in Figure 1. Tuning the acene core size was deemed as a particularly interesting molecular engineering strategy, given that electron polaron binding energies and hence the charge carrier transport abilities of organic semiconductors are dependent on the size and rigidity of the constituent acene core, and the length of the acene itself can tune the LUMO by varying the spacing along the backbone of the electron withdrawing lactam units. ${ }^{27}$
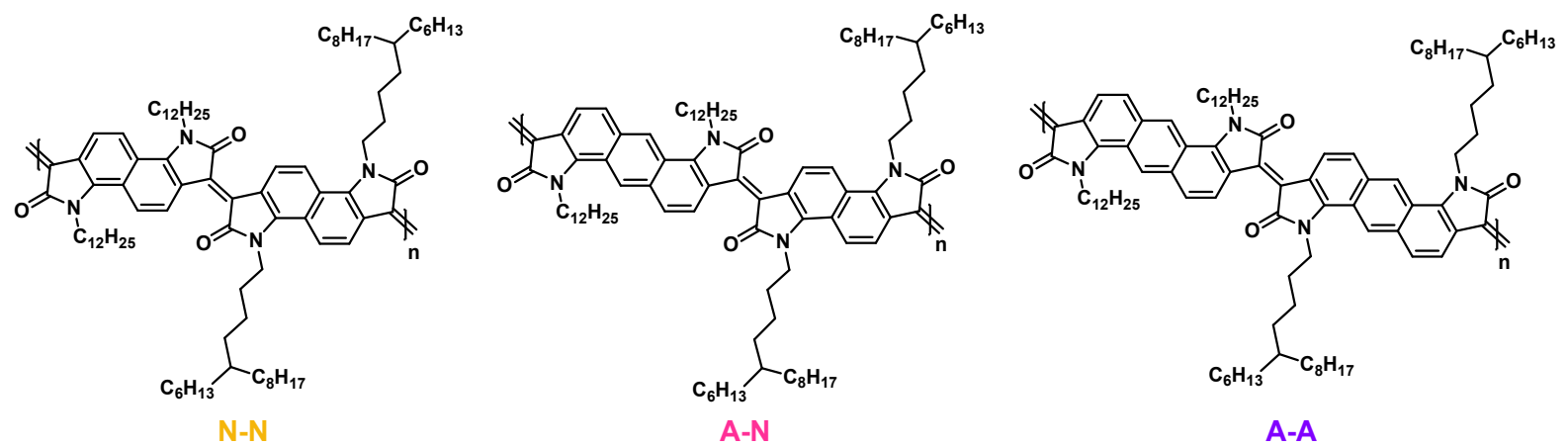

A-A

Figure 1. Chemical structures of the rigid polymers investigated within this study.

\section{Polymer Synthesis}


The syntheses of the bis-oxindole and bis-isatin monomers followed a general strategy reported in the literature. ${ }^{26}$ For the bis-oxindole monomers, this involved acylation of the corresponding aryl diamine with lauroyl chloride, followed by reduction of the resulting amide with lithium aluminum hydride to the analogous amine. The amine containing aryl species was subsequently reacted with chloroacetyl chloride followed by an intramolecular Heck-type cyclisation to yield the desired bis-oxindole. A more detailed explanation of the synthetic procedures can be found in the supporting information (S.I.).

The synthesis of the bis-isatin monomers on the other hand was based on a Martinet isatin approach. Specifically, this involved reacting the same aryl diamine starting material employed for the bis-oxindole synthesis with diethyl ketomalonate followed by alkylation with the desired alkyl iodide. The polymers $\mathbf{N}-\mathbf{N}, \mathbf{A}-\mathbf{N}$ and A-A were ultimately synthesized by acid catalyzed aldol polycondensation between the enolizable carbonyl unit of the bis-oxindole monomers and the electrophilic carbonyl unit of the corresponding bis-isatin co-monomers. This polymerization approach offers numerous advantages compared to the traditional transition-metal catalyzed one, as it does not necessitate the use of expensive transition metal catalysts such as palladium and it does not require the formation and use of highly toxic organostannane coupling partners, such as those required for Stille cross-couplings. ${ }^{24,26}$ Ultimately, the resulting polymers were obtained in high molecular weights, with $\mathrm{M}_{\mathrm{n}}>50 \mathrm{kDa}$, which is often a pre-requisite for high-performance materials in organic electronic applications. ${ }^{28,29}$

\section{Polymer Characterization}

The energy levels of the polymers were investigated through a combination of low energy inverse photoemission spectroscopy (LE-IPES), ultraviolet photoelectron spectroscopy (UPS) and UV-Vis spectroscopy and are summarized in Table 1. LE-IPES, UPS and UV-vis 
spectroscopy were employed to determine the electron affinity (EA), ionization potential (IP) and optical gaps $\left(E_{g}\right)$ of the polymers respectively, see Figure S1 and Figure 2. The UPS and LE-IPES spectra onsets were assigned as $2 \sigma$ from the leading-edge Gaussian fitted peak. ${ }^{30-32}$ As shown in Table 1, all three polymers exhibited a similarly small $\mathrm{E}_{\mathrm{g}}$, while the EA of the polymers became progressively shallower, moving from $-3.94 \mathrm{eV}$ to $-3.72 \mathrm{eV}$, upon increasing the size of the aromatic core in the polymer backbone, i.e. upon going from an all naphthalene to a mixed naphthalene-anthracene to an all anthracene polymer backbone. This was attributed to the effective dilution of the electron withdrawing properties of the four lactam moieties per polymer repeat unit, consequently rendering the larger anthracene containing systems less electron deficient compared to their naphthalene counterparts. The IPs extracted from the HOMO region of the UPS spectra for the three semiconductors displayed the same trend as their EAs, with the all naphthalene-based polymer, $\mathbf{N}-\mathbf{N}$, showing the largest IP and the all anthracene-based polymer, A-A, possessing the smallest IP, indicating that the orbital electron densities are strongly determined by the electron withdrawing properties of the lactam groups.

Table 1. Summary of the energy levels and optical properties of the polymers under investigation.

\begin{tabular}{lcccc}
\hline Polymer & ${\mathbf{I P ~}(\mathbf{e V})^{a}}$ & $\mathbf{E A}(\mathbf{e V})^{b}$ & $\lambda_{\text {max,film }}(\mathbf{n m})$ & $\mathbf{E}_{\mathbf{g}}(\mathbf{e V})^{c}$ \\
\hline $\mathrm{N}-\mathrm{N}$ & -5.34 & -3.94 & 915 & 1.04 \\
$\mathrm{~A}-\mathrm{N}$ & -5.29 & -3.83 & 1039 & 1.03 \\
$\mathrm{~A}-\mathrm{A}$ & -5.18 & -3.72 & 1064 & 1.03 \\
${ }^{a}$ Extracted from the HOMO & region of the UPS spectrum. \\
${ }^{b}$ Determined from LE-IPES. ${ }^{c}$ Calculated from the onset of \\
absorption in thin film samples.
\end{tabular}


UV-Vis-NIR absorption spectroscopy was conducted on both pristine and doped thinfilm samples of the three semiconductors. The corresponding UV-Vis absorption spectra can be found in Figure 2, where in the pristine state, all polymers exhibited two main absorption peaks, one with an absorption onset around $1200 \mathrm{~nm}$ and one with an absorption onset around $590 \mathrm{~nm}$. Given that the higher energy transition occurred at energies that were close to two times in magnitude compared to the lower energy absorption, the two transitions were attributed to the $S_{0}$ to $S_{1}$ and $S_{0}$ to $S_{2}$ optical transitions respectively. The UV-Vis absorption profiles of the three semiconductors also showed a significant difference in terms of vibronic features. In fact, the absorption profile of $\mathbf{N}-\mathbf{N}$ displayed a maximum absorption wavelength $\left(\lambda_{\max , \text { film }}\right)$ at $915 \mathrm{~nm}$ and no well-resolved fine structure. On the other hand, both anthracene containing polymers, A-N and A-A, demonstrated more resolved fine structures with $\mathbf{A}-\mathbf{N}$ and A-A displaying low energy shoulders, which in turn resulted in a red-shift of their thin film absorption maxima to $1039 \mathrm{~nm}$ and $1064 \mathrm{~nm}$ respectively.

Addition of $25 \mathrm{~mol} \%$ dopant, specifically 4-(2,3-dihydro-1,3-dimethyl-1Hbenzimidazol-2-yl)-N,N-dimethylbenzenamine (N-DMBI), was used to gain insights into the doping process of the polymers. N-DMBI was chosen to solution dope the polymers, thus rendering the doping process compatible with low cost roll-to-roll printing techniques. ${ }^{33}$ As highlighted in Figure 2, introduction of N-DMBI in each polymer film resulted in the appearance of a new absorption feature with an absorption maximum at approximately 1900 $\mathrm{nm}$, which was attributed to the polaronic species of the various semiconductors. Although NDMBI could successfully dope each of the three polymers, the intensity of the longer wavelength absorption feature relative to the corresponding pristine polymer absorption varied significantly across the polymers, see Figure 2 d-f. N-N and A-N gave more intense polaron transitions compared to A-A, consequently indicating a lower degree of doping in A-A under the same doping conditions. This was ascribed to the lower lying LUMOs of $\mathbf{N}-\mathbf{N}$ and $\mathbf{A}-\mathbf{N}$, 
rendering the doping process thermodynamically more favorable. UV-Vis-NIR absorption studies thus highlighted that varying the size of the central aryl core in the conjugated polymer backbone had a strong impact on the doping level that could be achieved by the polymers.
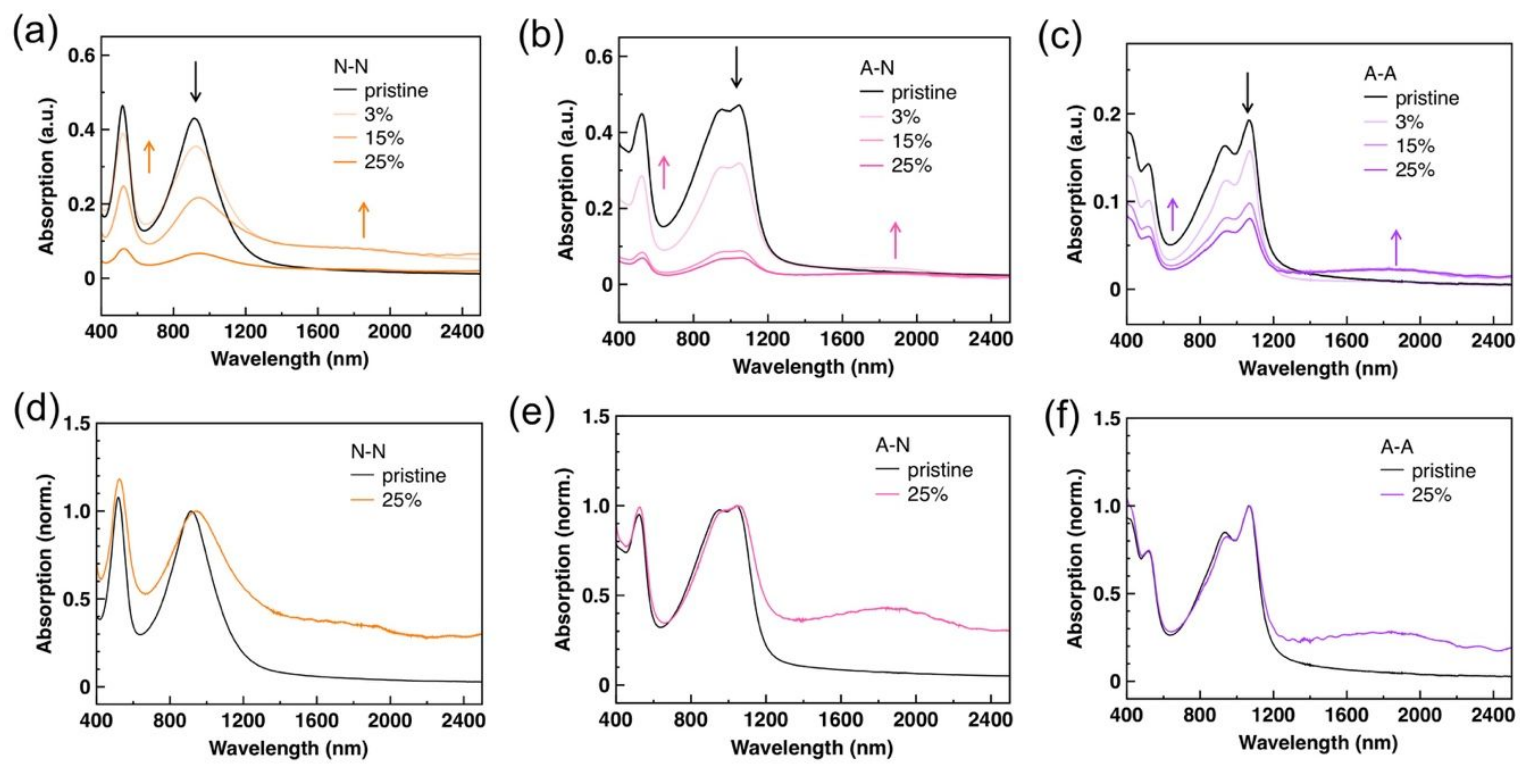

Figure 2. UV-Vis-NIR absorption spectra of the pristine and doped a) N-N, b) A-N c) A-A, and normalized doped absorption spectra of d) $\mathbf{N}-\mathbf{N}$, e) A-N and f) A-A.

The thermal properties of the polymers were investigated by both thermogravimetric analysis (TGA) and differential scanning calorimetry (DSC). The TGA and DSC traces of $\mathbf{N}-$ N, A-N and A-A can be found in Figures S2-S5 in the S.I. All of the polymers displayed excellent thermal stabilities showing no signs of decomposition up to $360^{\circ} \mathrm{C}$, thus suggesting their suitability for thermoelectric applications. Moreover, the DSC traces recorded for all of the polymers were featureless, highlighting the absence of any significant morphological changes upon heating or cooling of the samples.

To gain further insights into the morphological properties and the relative degree of order of the various polymers, grazing-incidence wide-angle X-ray scattering (GIWAXS) was 
performed on polymer films cast on silicon substrates. The two-dimensional scattering patterns and 1-dimensional line profiles are illustrated in Figure 3.

(a)
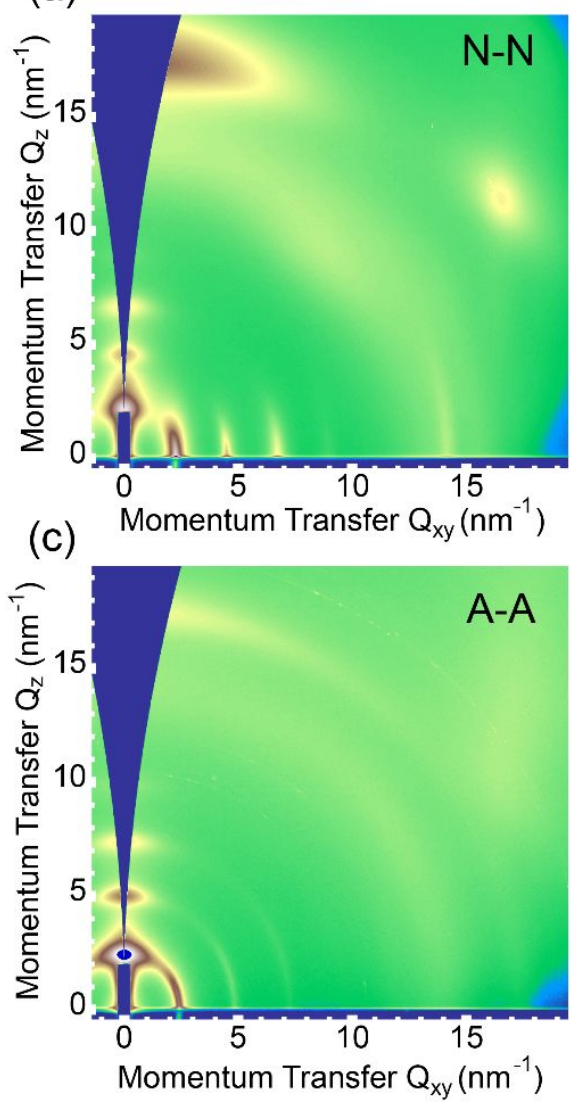

(b)
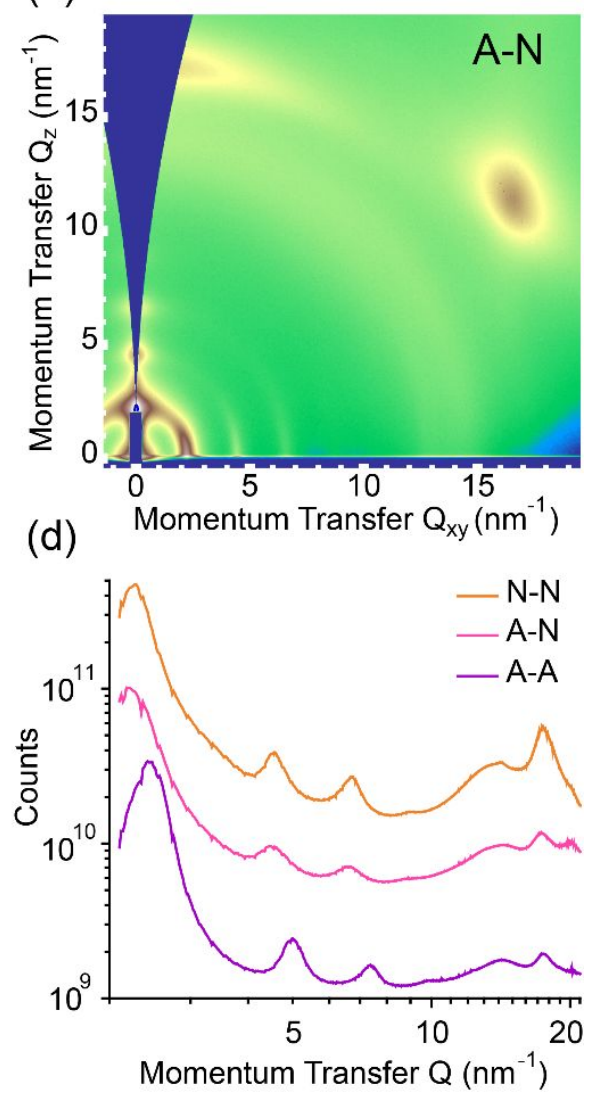

Figure 3. GIWAXS data of the three polymers: two-dimensional scattering patterns of a) N$\mathbf{N}$, b) A-N, c) A-A. (d) Comparison of the azimuthally integrated one-dimensional scattering profiles recorded for the three polymers. Profiles offset vertically for clarity.

As shown in Figure 3, all polymers exhibited a semi-crystalline microstructure with both lamellar stacking and $\pi$-stacking peaks evident. Despite having identical side chains, the lamellar stacking peaks of the three polymers appeared at slightly different values of $Q$, corresponding to different lamellar stacking distances. For N-N the (100) peak appeared at 2.29 $\mathrm{nm}^{-1}$, corresponding to a d-spacing of $2.75 \mathrm{~nm}$. The (100) peak of A-N in comparison occurred at $2.25 \mathrm{~nm}^{-1}$ (corresponding to a d-spacing of $2.80 \mathrm{~nm}$ ) while the (100) peak of A-A was 
recorded at $2.44 \mathrm{~nm}^{-1}$ (corresponding to a d-spacing of $2.58 \mathrm{~nm}$ ). This indicates that the A-A polymer had the shortest lamellar stacking distance which is likely afforded by the longer backbone repeat unit distance, which in turn may allow for some degree of interdigitation or space-filling of the side chains. The films also exhibited differences in texture: the (100) peak of N-N appeared largely in-plane (along $Q_{x y}$ ) while the (100) peak of A-A appeared predominantly out-of-plane (along $Q_{z}$ ). The A-N polymer shows a somewhat intermediate character suggesting a transition from a predominantly face-on stacking for $\mathbf{N}-\mathbf{N}$ to a predominantly edge-on stacking for A-A. While this could be attributed to an intrinsic material property, an alternative possibility, corroborating previous literature reports, is that the progressively lower solubility of the polymers in the processing solvent upon acene core expansion, leads to pre-aggregation in solution, forming lamella sheets, which deposit in the plane of the substrate, with a preferential edge-on deposition of the backbone, orthogonal to the lamella. ${ }^{34,35}$

The $\pi-\pi$ stacking peak for the three materials occurred at $17.4 \mathrm{~nm}^{-1}$ for $\mathbf{N}-\mathbf{N}, 17.3 \mathrm{~nm}^{-1}$ for $\mathbf{A}-\mathbf{N}$, and $17.6 \mathrm{~nm}^{-1}$ for $\mathbf{A}-\mathbf{A}$, corresponding to similar $\pi-\pi$ stacking distances of $\sim 0.36 \mathrm{~nm}$ for all polymers. It is noted that the A-N polymer exhibits broader lamellar stacking peaks indicating a smaller crystallite size. In particular the coherence lengths for the three polymers based on analysis of the full-width-at-half-maximum (FWHM) of the (100) peak were 34.5 nm, $19.2 \mathrm{~nm}$, and $29.5 \mathrm{~nm}$ for $\mathbf{N}-\mathbf{N}, \mathbf{A}-\mathbf{N}$, and $\mathbf{A}-\mathbf{A}$, respectively.

To complement the experimentally recorded data, density functional theory (DFT) calculations were carried out on tetramers of the various polymers employing an optimally tuned $\omega$ B97X-D functional and $6-31 \mathrm{G}^{*}$ as the basis set. The DFT optimized structures of the three tetramers are shown in Figure S9, S11 and S13 in the S.I. As is common in the field, the long solubilizing alkyl chains were replaced by the shorter methyl groups to simplify the simulations. 
First, geometry optimizations of the tetramers were performed, revealing that the anti configuration, i.e. the one in which the carbonyl moieties of neighboring bis-isoindigo units are oriented in opposite directions, to be the thermodynamically most favorable configuration, see Figure S6 in the S.I. Closer evaluation of the $120-180^{\circ}$ region of the torsional potential energy surface, see Figure 4, showed an energetic minimum at $165^{\circ}$, thus indicating the almost planar configuration assumed by the conjugated polymer backbone for the three polymers. Also note the relatively shallow torsional potential energy surface (PES) incurred at $165^{\circ}$ by each of the three polymers, which enables dihedral angle fluctuations of $\pm 15^{\circ}$ at room temperature. This feature, in combination with intermolecular packing interactions between neighboring polymer chains is likely to enable the polymers to assume an even more planar configuration in the solid state, thus benefiting orbital overlap and in turn also polaron delocalization. These findings were not only analogous to computational simulations of alternative ladder-type polymers, but were also in agreement with previous crystallographic data collected on substituted isoindigos, in which the dihedral angle across the carbon-carbon double bond linking the two oxindole rings was found to be around $22^{\circ} \cdot{ }^{25,26,36}$ The relatively small dihedral angles obtained around the carbon-carbon bonds connecting neighboring bis-isoindigo units was attributed to their double bond character. The non-fully planar nature of this bond on the other hand was ascribed to steric interactions between the carbonyl moiety located on one aryl core and the nearest hydrogen atoms of the adjacent acene core. In fact, the average oxygenhydrogen distance measured around $2.15 \AA$, a value that is significantly smaller than the sum of the van der Waals radii of oxygen $(1.52 \AA)$ and hydrogen $(1.20 \AA)$, thus also being in agreement with literature data on related ladder-type polymers. ${ }^{25}$ Ultimately, the combination of the highly rigid aryl cores possessed by the various tetramers and the relatively planar polymer backbones are likely to be beneficial for the intramolecular charge transport of these materials. 
The torsional potentials of the radical anion states of the three polymers were also identical, and only slight differences to the neutral polymers were observed. Rotation to lower dihedral angles becomes slightly easier (shallower PES) while rotation to increasing dihedral angle (more planar) becomes slightly more difficult. However, these differences were very marginal, and overall, the polymers retained their rigidity when in the anionic state.

Single point DFT calculations on the optimized geometries were performed to determine the MO distribution of the neutral polymer LUMO and the singly occupied molecular orbital (SOMO) of the anionic polymer over the eight acene fragments comprising the tetramers of the various polymers. As shown in Figure 4 the neutral polymer LUMOs were primarily distributed on the central four acene units of all the polymers. The slight difference in the shape of the MO contribution trace of A-N compared to the ones of $\mathbf{N}-\mathbf{N}$ and A-A was due to the mixed naphthalene and anthracene cores present within the A-N polymer. As seen in Figure 4, the introduction of two different acene cores resulted in an asymmetric LUMO distribution of the A-N polymer, which in turn were due to the stronger contributions from the lactam groups in the central two naphthalene cores to the LUMO in comparison to those in the anthracene cores. These findings therefore indicate that the naphthalene bis-oxindole unit had a more electron deficient character compared to its anthracene counterpart, thus corroborating the findings from UPS and LE-IPES.

We envisaged the SOMO of the anionic polymer distribution to be of particular interest given its analogy to the electron polaron of the corresponding polymers. The MO distribution of the anionic polymer SOMO showed more localized character than the neutral LUMO, but significant differences were observed across the three polymers, see Figure 4, with the N-N polymer showing the broadest distribution and the A-A polymer the narrowest one. To better quantify the width of the MO distribution curve and use it as an indicator of the delocalization of the anionic polymer SOMO and hence polaron, we calculated the full width half maximum 
(FWHM) of the MO distribution curves. The FWHM computed for the $\mathbf{N}-\mathbf{N}, \mathbf{A}-\mathbf{N}$ and $\mathbf{A}-\mathbf{A}$ polymers were 2.4 fragments, 2.3 fragments and 2.0 fragments respectively, thus revealing that the anionic polymer SOMO of $\mathbf{N}-\mathbf{N}$ to be the most delocalized and that of the A-A polymer to be the least delocalized.

Hirshfeld atomic charges of each atom of the neutral polymer were subtracted from the equivalent atomic charges of the anionic polymer, leading to a visualization of where the extra charge upon reduction was found along the polymer chain. Within the fragment analysis, there is strong agreement with the MO contributions. Plotting on an atom-by-atom basis, see Figure S16 in the S.I., shows that most of the charge is located on only a few atoms, although the same trends in charge distribution can be observed. Interestingly, the mixed A-N polymer shows two similar environments bearing similar amounts of charge. Most of this charge is stabilized by the bridging $\mathrm{C}-\mathrm{C}$ double bond carbon atoms between fragments, and the carbonyl groups, see Figure S17 in the S.I. The comparison between N-N and A-A is also interesting, in that the fragment approach suggests that the $\mathbf{N}-\mathbf{N}$ polymer has a greater degree of delocalization. Turning to a distance comparison, A-A shows similar delocalization lengths to $\mathbf{N}-\mathbf{N}$, but $\mathbf{N}-\mathbf{N}$ spreads the charge over more repeat units, and over more of the more stabilizing $\mathrm{C}=\mathrm{C}$ and $\mathrm{C}=\mathrm{O}$ groups. Thus, in addition to changes in energy levels relevant to electronic doping, the change from naphthalene to anthracene also spaces out the atoms capable of stabilizing the excess charge, leading to localization on only a few groups. Consequently, this suggested the polaron in the $\mathbf{N}-\mathbf{N}$ polymer to be the most delocalized and hence $\mathbf{N}-\mathbf{N}$ to be most suited towards thermoelectric applications, given that previous studies have indicated a strong relationship between polaron delocalization and electrical conductivity. ${ }^{16}$ 

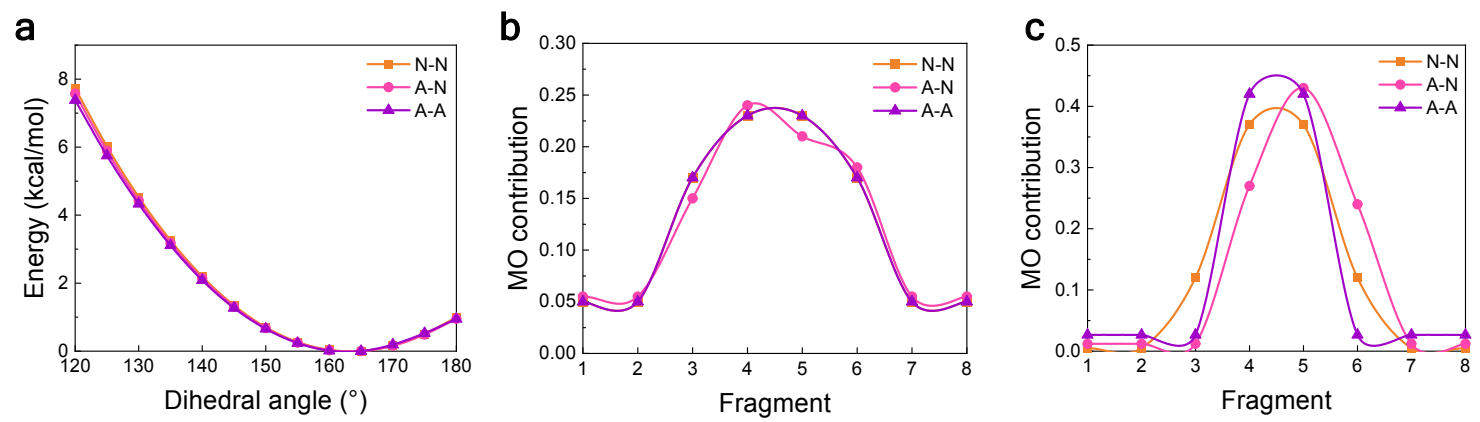

Figure 4. a) Torsional potentials around the center most $\mathrm{C}=\mathrm{C}$ bond in the neutral tetramers of N-N, A-N and A-A focusing on the $120-180^{\circ}$ region. Computed MO distributions of the b) neutral LUMO and c) anionic polymer SOMO for the tetramers of $\mathbf{N}-\mathbf{N}, \mathbf{A}-\mathbf{N}$ and $\mathbf{A}-\mathbf{A}$. The MO distribution traces of the neutral LUMO of $\mathbf{N}-\mathbf{N}$ and $\mathbf{A}-\mathbf{A}$ are overlapping.

Lastly, the energy levels and optical properties of the semiconductors were computed. The IPs calculated for N-N, A-N and A-A were -5.34 eV, -5.14 eV and -5.11 eV thus reflecting the trend recorded in the UPS measurements. A good fit between the experimental and computational trends obtained for the EAs of the polymers was also observed with the simulated EA for $\mathbf{N}-\mathbf{N}$ being the lowest $(-3.21 \mathrm{eV})$, for $\mathbf{A}-\mathbf{N}$ being in the middle $(-3.16 \mathrm{eV})$ and for A-A being the highest (-3.11 eV).

Having established the suitability of the series of ladder-type semiconducting polymers, their electrical conductivity, $\sigma$, which mathematically is given in equation 3 was investigated next.

$$
\sigma=n e \mu
$$

Where $n$ is the number density of charge carriers, $e$ the elementary charge and $\mu$ the charge carrier mobility. 
As highlighted in Figure 5 the electrical conductivity for each polymer is lowest in the absence of any N-DMBI. However, increasing the N-DMBI concentration resulted in an increase in the conductivity with $\sigma$ increasing over four orders of magnitude for each polymer. The maximum electrical conductivity $\left(\sigma_{\max }\right)$ recorded for $\mathbf{N}-\mathbf{N}, \mathbf{A}-\mathbf{N}$ and $\mathbf{A}-\mathbf{A}$ were $0.65 \mathrm{~S} \mathrm{~cm}^{-1}$, $0.26 \mathrm{~S} \mathrm{~cm}^{-1}$ and $0.018 \mathrm{~S} \mathrm{~cm}^{-1}$ respectively. The recorded trend in $\sigma_{\max }$ therefore is consistent with the trend in the electron mobility $\left(\mu_{\mathrm{e}}\right)$ for the three polymers, measured in organic field effect transistor (OFETs) devices that featured a top-gate bottom-contact (TGBC) architecture. The OFETs characterization data for each polymer can be found in Figure S18 the S.I. The electron mobilities recorded in the linear $\left(\mu_{\mathrm{e}, \text { lin }}\right)$ and saturation $\left(\mu_{\mathrm{e}, \mathrm{sat}}\right)$ regimes for the three polymers were $0.20 \mathrm{~cm}^{2} \mathrm{~V}^{-1} \mathrm{~s}^{-1}$ and $0.33 \mathrm{~cm}^{2} \mathrm{~V}^{-1} \mathrm{~s}^{-1}$ for $\mathbf{N}-\mathbf{N}, 0.15 \mathrm{~cm}^{2} \mathrm{~V}^{-1} \mathrm{~s}^{-1}$ and $0.23 \mathrm{~cm}^{2} \mathrm{~V}^{-}$ ${ }^{1} \mathrm{~s}^{-1}$ for $\mathbf{A}-\mathbf{N}$ and $0.05 \mathrm{~cm}^{2} \mathrm{~V}^{-1} \mathrm{~s}^{-1}$ and $0.07 \mathrm{~cm}^{2} \mathrm{~V}^{-1} \mathrm{~s}^{-1}$ for A-A respectively, thus highlighting that $\mathbf{N}-\mathbf{N}$ and A-N had comparable mobilities, while further increasing the acene core size was detrimental for charge carrier transport. Comparing the differences recorded in the $\sigma_{\max }$ with the differences recorded in the $\mu_{\mathrm{e}}$ for the polymers lead to the conclusion that the difference in the polymers' $\mu_{\mathrm{e}}$ is not sufficient to fully explain the difference in $\sigma_{\max }$. In fact, the larger difference in $\sigma_{\max }$ suggests that increasing the acene core size also results in lower charge carrier densities, $n$, generated during the doping process, which fully agrees with the stronger polaronic absorption recorded for the doped N-N film compared to the doped A-A film, see Figure 2d-f. Finally, the recoded $\sigma_{\max }$ trend also followed the trend in the simulated anionic polymer SOMO delocalization, whereby increasing the anionic polymer SOMO delocalization led to polymers with a higher $\sigma_{\max }$. This in turn further supported previous literature evidence of a correlation between the degree of delocalization of the electron polaron and the electrical conductivity of materials. ${ }^{16}$ 
a)

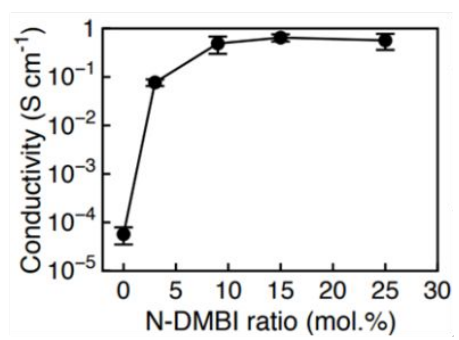

b)

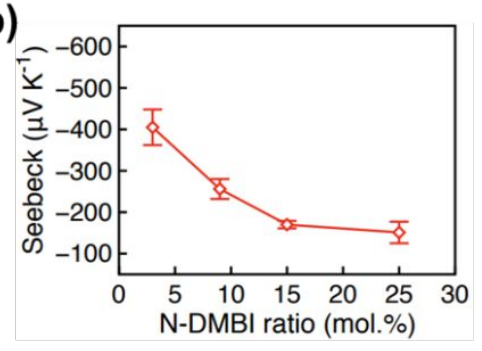

c)

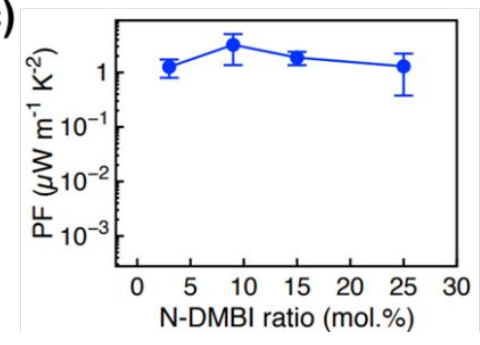

A-N
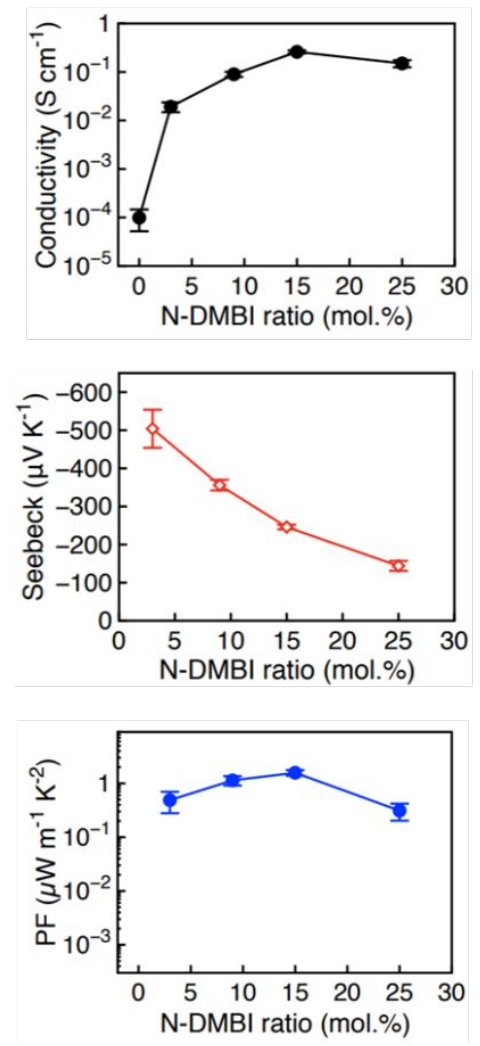

A-A
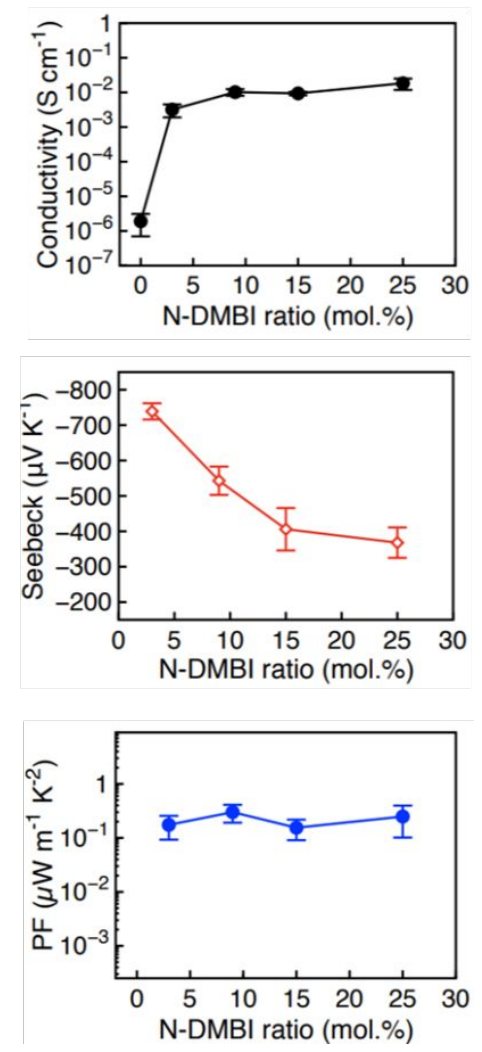

Figure 5. a) Electrical conductivities, b) Seebeck coefficients and c) power factors recorded for $\mathbf{N}-\mathbf{N}, \mathbf{A}-\mathbf{N}$ and $\mathbf{A}-\mathbf{A}$ as a function of N-DMBI ratio employed.

Next, the thermopower of the three semiconductors was measured. For all the three members of the series negative thermopower values were obtained, hence indicating that electrons were the majority carrier within each of the polymers. The Seebeck coefficient for N-N, A-N and A-A was highest at a dopant ratio of $3 \mathrm{~mol} \%$ and decreased progressively upon increasing the dopant ratio. At each N-DMBI ratio, N-N and A-A displayed the lowest and highest $S$ respectively.

As anticipated and highlighted in Figure 5, the evolution of $\sigma$ and $S$ as a function of dopant ratio followed an opposite trend, thus signifying that the maximum power factor for the polymers was obtained at a dopant concentration that would best balance the two quantities. 
The maximum PFs of the three semiconductors were recorded at different dopant concentrations with $\mathbf{N}-\mathbf{N}$ affording the highest PF of $3.2 \mu \mathrm{W} \mathrm{m} \mathrm{m}^{-1} \mathrm{~K}^{-2}$ at $9 \mathrm{~mol} \% \mathrm{~N}-\mathrm{DMBI}$ concentration, A-N a PF of $1.6 \mu \mathrm{W} \mathrm{m}^{-1} \mathrm{~K}^{-2}$ at $15 \mathrm{~mol} \% \mathrm{~N}-\mathrm{DMBI}$ content and A-A a PF of 0.25 $\mu \mathrm{W} \mathrm{m} \mathrm{m}^{-1} \mathrm{~K}^{-2}$ at $25 \%$ dopant ratio. The PF recorded for $\mathbf{N}-\mathbf{N}$ and $\mathbf{A}-\mathbf{N}$ are amongst the highest recorded for n-type OTE materials to date, while the PF recorded for A-A is still higher compared to the thoroughly studied and optimized N2200 polymer. ${ }^{12,15,17,19,20,25}$

Table 2. Organic field effect transistor and thermoelectric performance summary of the polymers under investigation.

\begin{tabular}{ccccc}
\hline Polymer & $\begin{array}{c}\boldsymbol{\mu}_{e, \text { lin }}{ }^{a} \\
\left(\mathbf{c m}^{2} \mathbf{V}^{-1} \mathbf{s}^{-1}\right)\end{array}$ & $\begin{array}{c}\boldsymbol{\mu}_{e, \text { sat }}{ }^{a} \\
\left(\mathbf{c m}^{\mathbf{2}} \mathbf{V}^{-1} \mathbf{s}^{-\mathbf{1}}\right)\end{array}$ & $\begin{array}{c}\boldsymbol{\sigma}_{\max } \\
\left(\mathbf{S ~ c m}^{-1}\right)\end{array}$ & $\begin{array}{c}\boldsymbol{P F}_{\text {max }} \\
\left(\boldsymbol{\mu} \mathbf{W ~ m}^{-1} \mathbf{K}^{-2}\right)\end{array}$ \\
\hline N-N & 0.20 & 0.33 & 0.65 & 3.2 \\
A-N & 0.15 & 0.23 & 0.26 & 1.6 \\
A-A & 0.05 & 0.07 & 0.018 & 0.25
\end{tabular}

${ }^{a}$ Obtained from TGBC OFET measurements.

\section{Conclusion}

In summary, three new n-type fused lactam semiconducting polymers only differing in the relative size of their constituent acene cores were synthesized for thermoelectric and transistor applications. Compared to traditional transition-metal catalyzed polymerization reactions for conjugated polymers, the materials developed herein were obtained via a convenient transition-metal free aldol polycondensation reaction, thus avoiding the use of expensive palladium-based catalysts and any highly toxic organometallic synthetic intermediates. TGA measurements indicated excellent thermal stability of each polymer, with none of the materials showing any sign of decomposition up to $360{ }^{\circ} \mathrm{C}$. Experimental and computational energy level analysis of the three polymers on the other hand demonstrated that 
reducing the central acene core size from two anthracene (A-A), to mixed naphthaleneanthracene $(\mathbf{A}-\mathbf{N})$ and two naphthalene cores $(\mathbf{N}-\mathbf{N})$ progressively resulted in larger EAs, thereby leading to an increasingly more favorable and efficient doping process when employing N-DMBI as the dopant. In parallel, both OFET mobility data and DFT simulations also suggested the benefits of acene core contraction on the thermoelectric performance, with the N-N polymer affording the highest electron mobility and the most delocalized electron polaron and the A-A polymer incurring the lowest values. Ultimately, these concepts were clearly reflected in the thermoelectric performance data recorded for the polymers with $\mathbf{N}-\mathbf{N}$, A-N and A-A affording maximum electrical conductivities and power factors of $0.65 \mathrm{~S} \mathrm{~cm}^{-1}$ and $3.2 \mu \mathrm{W} \mathrm{m} \mathrm{m}^{-1} \mathrm{~K}^{-2}, 0.26 \mathrm{~S} \mathrm{~cm}^{-1}$ and $1.6 \mu \mathrm{W} \mathrm{m} \mathrm{K}^{-1}$ and $0.018 \mathrm{~S} \mathrm{~cm}^{-1}$ and $0.25 \mu \mathrm{W} \mathrm{m} \mathrm{K}^{-2}$ when doped with N-DMBI respectively. The PF recorded for $\mathbf{N}-\mathbf{N}$ and $\mathbf{A}-\mathbf{N}$ are thus amongst the highest reported in the literature for n-type polymers. Importantly, the results reported in this study highlight that modulating the size of the central acene ring is a highly effective molecular design strategy to optimize the thermoelectric performance thus also providing new insights into the molecular design guidelines for the next generation of high-performance ntype materials for thermoelectric applications.

\section{Acknowledgements}

The authors acknowledge generous funding from KAUST for financial support. The research reported in this publication was sponsored by funding from King Abdullah University of Science and Technology Office of Sponsored Research (OSR) under awards no. OSR-2018CARF/CCF-3079, no. OSR-2015-CRG4-2572 and OSR-4106 CPF2019. We acknowledge EC FP7 Project SC2 (610115), EC H2020 (643791), and EPSRC Projects EP/G037515/1, EP/M005143/1, and EP/L016702/1. This work was performed in part at the SAXS/WAXS 
beamline at the Australian Synchrotron, part of ANSTO. ${ }^{37}$ S.F. acknowledges financial support from the Swedish Research Council (2016-03979), ÅForsk (18-313, 19-310), Olle Engkvists Stiftelse (204-0256), and the Advanced Functional Materials Center at Linköping University (2009-00971).

\section{References}

1 Kroon, R.; Mengistie, D. A.; Kiefer, D.; Hynynen, J.; Ryan, J. D.; Yu, L.; Müller, C. Chem. Soc. Rev. 2016, 45, 6147-6164.

2 Zhang, Q.; Sun, Y.; Xu, W.; Zhu, D. Adv. Mater. 2014, 26, 6829-6851.

3 Bell, L. E. Science. 2008, 321, 1457-1461.

4 Wüsten, J.; Potje-Kamloth, K. J. Phys. D. Appl. Phys. 2008, 41, 135113.

5 Zebarjadi, M.; Esfarjani, K.; Dresselhaus, M. S.; Ren, Z. F.; Chen, G. Energy Environ. Sci. 2012, 5, 5147-5162.

6 Russ, B.; Glaudell, A.; Urban, J. J.; Chabinyc, M. L.; Segalman, R. A. Nat. Rev. Mater. 2016, $1,16050$.

7 Goel, M.; Heinrich, C. D.; Krauss, G.; Thelakkat, M. Macromol. Rapid Commun. 2019, 40, 1800915.

$8 \quad$ Yi, C.; Wilhite, A.; Zhang, L.; Hu, R.; Chuang, S. S. C.; Zheng, J.; Gong, X. ACS Appl. Mater. Interfaces 2015, 7, 8984-8989.

$9 \quad$ Fan, Z.; Li, P.; Du, D.; Ouyang, J. Adv. Energy Mater. 2017, 7, 1602116.

10 Wang, X.; Zhang, X.; Sun, L.; Lee, D.; Lee, S.; Wang, M.; Zhao, J.; Shao-Horn, Y.; Dincă, M.; Palacios, T.; Gleason, K. K. Sci. Adv. 2018, 4, eaat5780. 
11 Patel, S. N.; Glaudell, A. M.; Kiefer, D.; Chabinyc, M. L. ACS Macro Lett. 2016, 5, $268-272$.

12 Yan, X.; Xiong, M.; Li, J. T.; Zhang, S.; Ahmad, Z.; Lu, Y.; Wang, Z. Y.; Yao, Z. F.; Wang, J. Y.; Gu, X.; Lei, T. J. Am. Chem. Soc. 2019, 141, 20215-20221.

13 Wang, Y.; Nakano, M.; Michinobu, T.; Kiyota, Y.; Mori, T.; Takimiya, K. Macromolecules 2017, 50, 857-864.

14 Liu, J.; Shi, Y.; Dong, J.; Nugraha, M. I.; Qiu, X.; Su, M.; Chiechi, R. C.; Baran, D.; Portale, G.; Guo, X.; Koster, L. J. A. ACS Energy Lett. 2019, 4, 1556-1564.

15 Shi, K.; Zhang, F.; Di, C. A.; Yan, T. W.; Zou, Y.; Zhou, X.; Zhu, D.; Wang, J. Y.; Pei, J. J. Am. Chem. Soc. 2015, 137, 6979-6982.

16 Naab, B. D.; Zhang, S.; Vandewal, K.; Salleo, A.; Barlow, S.; Marder, S. R.; Bao, Z. Adv. Mater. 2014, 26, 4268-4272.

17 Wang, S.; Sun, H.; Erdmann, T.; Wang, G.; Fazzi, D.; Lappan, U.; Puttisong, Y.; Chen, Z.; Berggren, M.; Crispin, X.; Kiriy, A.; Voit, B.; Marks, T. J.; Fabiano, S.; Facchetti, A. Adv. Mater. 2018, 30, 1801898.

18 Wang, S.; Sun, H.; Ail, U.; Vagin, M.; Persson, P. O. Å.; Andreasen, J. W.; Thiel, W.; Berggren, M.; Crispin, X.; Fazzi, D.; Fabiano, S. Adv. Mater. 2016, 28, 10764-10771.

19 Kiefer, D.; Giovannitti, A.; Sun, H.; Biskup, T.; Hofmann, A.; Koopmans, M.; Cendra, C.; Weber, S.; Anton Koster, L. J.; Olsson, E.; Rivnay, J.; Fabiano, S.; McCulloch, I.; Müller, C. ACS Energy Lett. 2018, 3, 278-285.

20 Liu, J.; Qiu, L.; Alessandri, R.; Qiu, X.; Portale, G.; Dong, J. J.; Talsma, W.; Ye, G.; Sengrian, A. A.; Souza, P. C. T.; Loi, M. A.; Chiechi, R. C.; Marrink, S. J.; Hummelen, J. C.; Koster, L. J. A. Adv. Mater. 2018, 30, 1704630. 
21 Schlitz, R. A.; Brunetti, F. G.; Glaudell, A. M.; Miller, P. L.; Brady, M. A.; Takacs, C. J.; Hawker, C. J.; Chabinyc, M. L. Adv. Mater. 2014, 26, 2825-2830.

22 Di Nuzzo, D.; Fontanesi, C.; Jones, R.; Allard, S.; Dumsch, I.; Scherf, U.; Von Hauff, E.; Schumacher, S.; Da Como, E. Nat. Commun. 2015, 6, 6460.

23 Liu, J.; Ye, G.; van der Zee, B.; Dong, J.; Qiu, X.; Liu, Y.; Portale, G.; Chiechi, R. C.; Koster, L. J. A. Adv. Mater. 2018, 30, 1804290.

24 Lee, J.; Kalin, A. J.; Yuan, T.; Al-Hashimi, M.; Fang, L. Chem. Sci. 2017, 8, 2503-2521.

25 Lu, Y.; Yu, Z. Di; Zhang, R. Z.; Yao, Z. F.; You, H. Y.; Jiang, L.; Un, H. I.; Dong, B. W.; Xiong, M.; Wang, J. Y.; Pei, J. Angew. Chemie - Int. Ed. 2019, 58, 11390-11394.

26 Onwubiko, A.; Yue, W.; Jellett, C.; Xiao, M.; Chen, H. Y.; Ravva, M. K.; Hanifi, D. A.; Knall, A. C.; Purushothaman, B.; Nikolka, M.; Flores, J. C.; Salleo, A.; Bredas, J. L.; Sirringhaus, H.; Hayoz, P.; McCulloch, I. Nat. Commun. 2018, 9, 416.

27 Coropceanu, V.; Cornil, J.; da Silva Filho, D. A.; Olivier, Y.; Silbey, R.; Brédas, J. L. Chem. Rev. 2007, 107, 926-952.

28 Pron, A.; Gawrys, P.; Zagorska, M.; Djurado, D.; Demadrille, R. Chem. Soc. Rev. 2010, $39,2577-2632$.

29 Kline, R. J.; McGehee, M. D.; Kadnikova, E. N.; Liu, J.; Fréchet, J. M. J. Adv. Mater. 2003, 15, 1519-1522.

30 Han, W.; Yoshida, H.; Ueno, N.; Kera, S. Appl. Phys. Lett. 2013, 103.

31 Yoshida, H. J. Phys. Chem. C 2014, 118, 24377-24382.

32 Yoshida, H. Rev. Sci. Instrum. 2014, 85, 016101.

33 Wei, P.; Oh, J. H.; Dong, G.; Bao, Z. J. Am. Chem. Soc. 2010, 132, 8852-8853. 
34 DeLongchamp, D. M.; Vogel, B. M.; Jung, Y.; Gurau, M. C.; Richter, C. A.; Kirillov, O. A.; Obrzut, J.; Fischer, D. A.; Sambasivan, S.; Richter, L. J.; Lin, E. K. Chem. Mater. 2005, 17, 5610-5612.

35 Zhao, L. H.; Png, R. Q.; Zhuo, J. M.; Wong, L. Y.; Tang, J. C.; Su, Y. S.; Chua, L. L. Macromolecules 2011, 44, 9692-9702.

36 Voronina, Y. K.; Krivolapov, D. B.; Bogdanov, A. V.; Mironov, V. F.; Litvinov, I. A.s J. Struct. Chem. 2012, 53, 413-416.

37 Kirby, N. M.; Mudie, S. T.; Hawley, A. M.; Cookson, D. J.; Mertens, H. D. T.; Cowieson, N.; Samardzic-Boban, V. J. Appl. Crystallogr. 2013, 46, 1670-1680. 